\title{
MAGNETIC FIELD CONFIGURATION IN H $\alpha$ FLARE LOOPS AND FLARING ARCHES
}

\author{
ARVIND BHATNAGAR AND NANDITA SRIVASTAVA \\ Udaipur Solar Observatory \\ Udaipur -313001 INDIA
}

\begin{abstract}
It is concluded from the observations of $\mathrm{H} \alpha$ flaring loops and flaring arches that these are two entirely different phenomena. From the reconstructed shape of flaring loops, it is suggested that the magnetic field lines revert to potential field configuration from sheared non-potential state.
\end{abstract}

\section{INTRODUCTION}

Major two-ribbon (TR) flares are known to be associated with a series of loops. These loops generally appear in $\mathrm{H} \alpha$ soon after the maximum phase of the flare. In the literature, these were referred as post-flare loops or more recently, simply as flare-loops. The shape and the orientation of the flare loops delineate the magnetic field configuration in the corona. Hence, a precise knowledge of the orientation of the flare loops would reveal the magnetic field configuration over the active region in the corona. With this objective, we have analysed two cases of flare loops associated with the TR flares observed on May 13, 1981 in Hale region 17644 and on June 4, 1991 in NOAA region 6659 (Fig. 1a and $1 \mathrm{~b}$ ). To compare the flare loops with an entirely different class of loops, we have included in this study, two remarkable events observed on March 6 and 7, 1991 in NOAA region 6538 (Fig. 2a and $2 b$ ). These events do have 'loop-like' appearance but are physically and morphologically quite different. In this case the $\mathrm{H} \alpha$ mass ejection takes place, perhaps during the initial or the maximum phase, from one footpoint in the chromosphere to another, separated by a large distance. The phenomenon lasts for only a few minutes, while the flare loops may continue for several hours after the flare maxima.

To determine the true shape and orientation of the loops on the solar surface, it is necessary to reconstruct the sky plane observations. This has been done using the geometrical method described by Loughhead et al. (1983). The method is based on three assumptions that (i) the central axis of the loop lies in a plane (ii) its footpoints can be located and (iii) it is symmetrical in its own plane and tilted away from the normal to the line joining the footpoints (Loughhead et al. 1984). To investigate which type of magnetic field configuration prevail in the flare loops, we have compared the geometrically reconstructed flare loops with the two proposed magnetic field line models: (a) the simple, lowest magnetic energy state of potential field configuration or the 

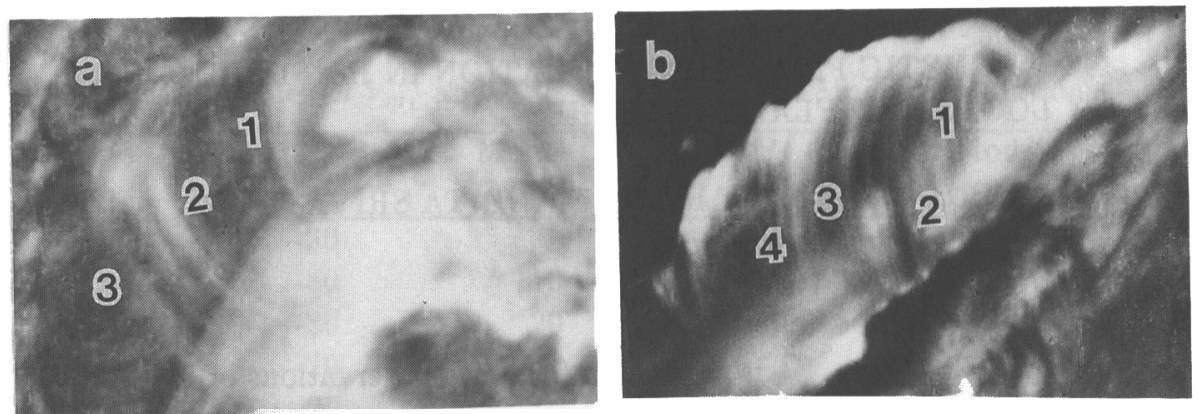

Figure 1: Flaring loops observed on (a).Max 13,.1981 (b) June 4,1991

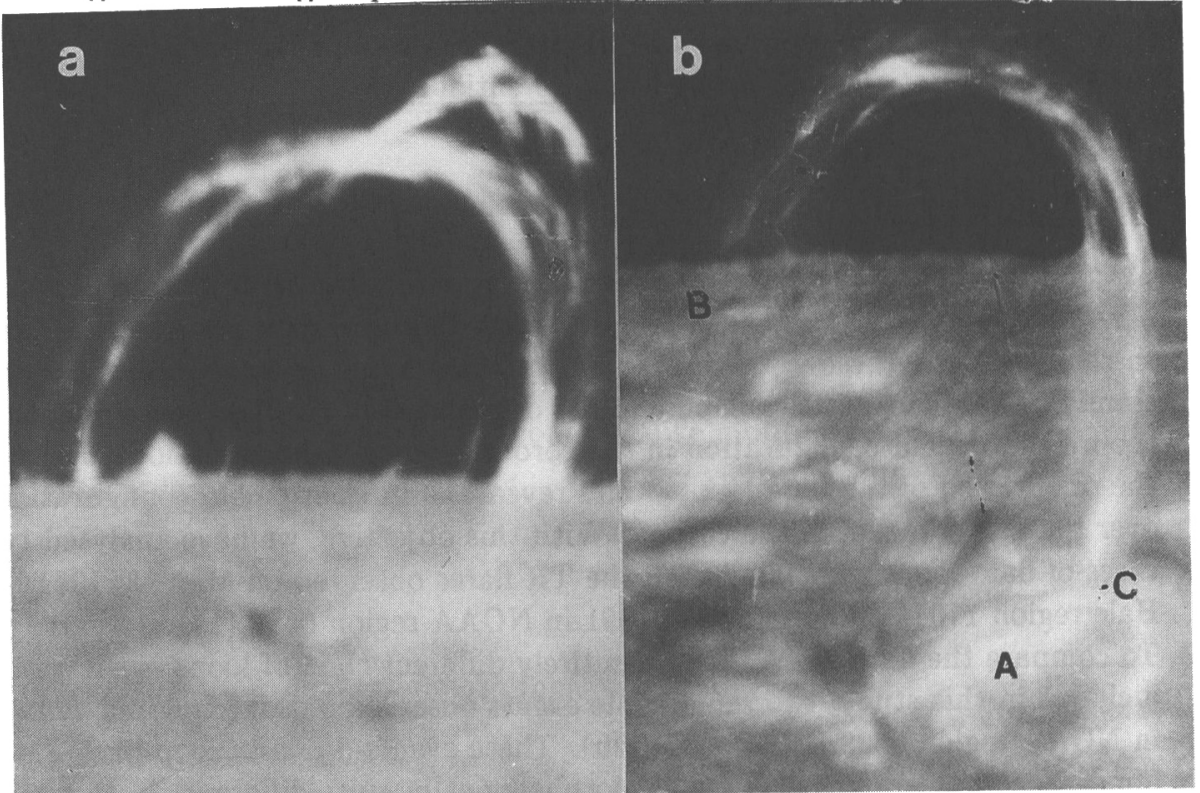

Figure 2: Flaring arches observed on (a) March 6, 1991 (b) March 7, 1991

dipole field. The dipole field lines are given by the equation

$$
r=(\delta+h) \cos ^{2} \chi
$$

where, $(r, \chi)$ is the polar coordinate of any point on the field line originating from a point dipole at a depth $\delta$ and $h$, the height of the apex of the loop. (b) the sheared, magnetic field line reconnection model due to Kopp and Pneuman (1976) or KP model. The field lines are given by the equation

$$
\frac{r^{3}+2\left(r_{0}+Z\right)^{3}}{\left(r_{0}+h\right)^{3}+2\left(r_{0}+Z\right)^{3}} \cos ^{2} \chi=\frac{r}{\left(r_{0}+h\right)}
$$

here, $(r, \chi)$ is any point on the field line, $h$, the height of the apex of the loop.The system of closed field lines is taken to extend from $r=r_{0}$ to $\mathrm{r}=r_{0}+\mathrm{Z}$, where $\mathrm{Z}$ increases with time. 


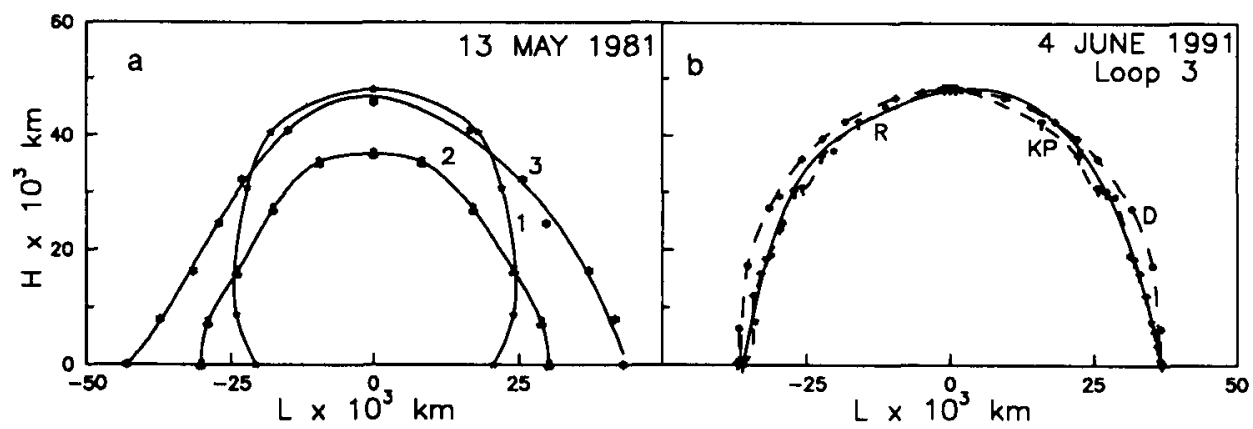

Figure 3: (a) Reconstructed loops of May 13, 1981 (b) Field lines due to dipole (D) and KP (KP) models compared with reconstructed loop (R)

\section{OBSERVATIONS, ANALYSIS AND DISCUSSION}

Flare loops of May 13, 1981 and June 4, 1991

We have selected three flare loops in the case of May 13, 1981 and four loops in the case of June 4, 1991, and reconstructed them geometrically. (Fig. 3a).

Several parameters characterising the orientation of the loops viz. the azimuthal angle of the loop plane $\alpha$, the inclination of the loop plane to the vertical $\beta$ and the tilt of the axis of symmetry of the loop, $\eta$, the distance between the footpoints and the height of all the loops were also computed. Using equations (1) and (2), the lines of force due to dipole and KP models were computed to compare with the central axis of the reconstructed loops. As an example, loop no. 3 of June 4 event is plotted in Fig. 3b. In the case of KP model, we have assumed three values i.e. $75,000,100,000,150,000 \mathrm{~km}$ for $Z$, the height of the highest loop. From the analysis of all the 7 flare loops, it is found that two loops (No. 2 and 3) of May 13 event and two loops (No. 2 and 3 ) of June 4 event, fit closely with the KP model. Loop No. 1 of May 13 fits well with the dipole field model while loops No. 1 and 4 of June 4 event lie in between the two. A similar result was obtained earlier by Loughhead et al. (1983), in the case of May 13, 1981 event. However, no explanation was given for such a disparity. We propose that the magnetic field is in a highly sheared or non-potential configuration, before and during the maximum phase of the TR flares. As the flare declines, the field reverts to low energy state or potential, dipole field configuration, hence, within the same set of flare loops, one finds loops corresponding to both, dipole and KP models.

Flaring Arches of March 6 and 71991

Homologous, huge mass ejections in the form of giant flare arches accompanied by very energetic flares were observed on March 6 and March 7, 1991 (Fig. 2a and $2 \mathrm{~b}$ ). These arches started just before the impulsive phase of the flare. On March 7, the flaring arches initiated from the point $A$, in the flare region and ended in a distant plage region B (Fig. 2b). It was found that the loops 
reached a maximum height of $120,000 \mathrm{~km}$ and the footpoints were separated by $110,000 \mathrm{~km}$. From the observed trajectory of the flaring arches, it is clearly seen that the ejected material followed a pre-existing magnetic field configuration in the corona. The remarkable similarity between the two cases (March 6 and 7) indicates that the field configuration was not modified after the flare of March 6 or if it changed, the coronal field identically reformed, as on the previous day. March 7 event, clearly displays the magnetic field reconnection taking place just above the low-lying flare loops, marked ' $\mathrm{C}$ '(Fig. 2b) from where the ejection of material took place into the corona.

\section{CONCLUSION}

Comparing the two different types of flare loops viz. the flare loops associated with TR flares, showing systematic downward material motion, with the huge flaring arches associated with compact, confined flares, it is concluded that these are two very different solar phenomena. The observed $\mathrm{H} \alpha$ flaring arches seem to be the optical counterpart of the giant arches observed in hard X-ray by HXIS, reported first by Svestka et al. (1982) while the flare loops are much lower level phenomena in which the material drains along the two 'legs' from the top of the loop.

\section{REFERENCES}

1. Loughhead, R.E., Wang, J.L. and Blows G., 1983, Ap. J., 274, 883

2. Loughhead, R.E., Chen Chuan Le and Wang, J. L., 1984, Solar Phys., 92, 53

3. Kopp, R.A. and Pneuman, G. W., 1976, Solar Phys., 50, 85

4. Svestka, Z., Stewart, R. T., Hoyng, P., Van Tend, W.,Acton, L. W., Gabriel, A. H. 1982, Solar Phys., 75, 305 